\title{
Effects of Emulsifiers on Preparing Spherical Urea-Formaldehyde Paraffin Capsules Modified by $\beta$-Cyclodextrin for Energy Storage
}

\author{
Xin Yu, ${ }^{1}$ Zhanhua Huang, ${ }^{1,2}$ and Kaixuan $Y u^{1}$ \\ ${ }^{1}$ Key laboratory of Bio-based Material Science and Technology of Ministry of Education, Northeast Forestry University, \\ Harbin 150040, China \\ ${ }^{2}$ School of Renewable Natural Resources, Louisiana State University Agricultural Center, Baton Rouge, LA 70803, USA \\ Correspondence should be addressed to Zhanhua Huang; huangzh1975@163.com
}

Received 22 November 2013; Revised 12 January 2014; Accepted 12 January 2014; Published 24 February 2014

Academic Editor: Ming-Guo Ma

Copyright ( 2014 Xin Yu et al. This is an open access article distributed under the Creative Commons Attribution License, which permits unrestricted use, distribution, and reproduction in any medium, provided the original work is properly cited.

\begin{abstract}
Urea-formaldehyde (UF) paraffin capsules (B-UPCS) modified by $\beta$-cyclodextrin $(\beta$-CD) were successfully prepared with excellent energy storage capacity using different emulsifiers. B-UPCS were characterized by FT-IR, DSC SEM, optical microscopy, and laser particle size distribution analyzer. The effects of different emulsifiers and the stirring rate of emulsion on the microstructure, average particle diameter, and the content of paraffin were discussed in details. The results show that OP-10 for use with PCMs as emulsifier has a better emulsifying capacity, thermal stability, and mechanical stirring stability than SDBS for use with PCMs as emulsifier. As a result, the optimum dosage of OP-10 was $3 \%$ to $7 \%$, and the suitable stirring rate of emulsion was 7500 to $12500 \mathrm{rpm}$. At this time, the spherical particle diameter of B-UPCS was about $1.0 \mu \mathrm{m}$, the paraffin content was $78 \%$, and the enthalpy of B-UPCS can reach $161.0 \mathrm{~J} / \mathrm{g}$.
\end{abstract}

\section{Introduction}

Phase change materials (PCMs) have been gradually attracting attention of people as new types of energy storage media. PCMs can store or release energy during the physical phase change process. For solid-liquid PCMs and liquid-liquid PCMs, they require a package to control and regulate energy during the phase change process. Encapsulation technology for use with PCMs is one kind of effective ways for preparing the core-shell capsule energy storage materials. The capsule shell acts like a container for PCMs encapsulation to achieve different sizes package for PCMs [1]. PCMs can improve the energy efficiency and expand the diversification of energy in intelligent ways. As a result, it has become the focus of research on PCMs in recent decades.

There are various methods for preparing PCMs, such as in situ polymerization [2], interfacial polymerization [3], sol-gel method [4], and spray drying [5]. The shell materials of PCMs are mainly melamine resin [6-9] and urea-formaldehyde resin [10-12]. But these kinds of shell materials in the preparation process require a large amount of formaldehyde, which is known as teratogenic substance and easy to cause environmental problems and public safety issues. Therefore, it makes great senses to use less formaldehyde or formaldehydefree materials for preparing PCMs. At the same time, it is necessary to improve the crosslinked degree of melamine resin and urea-formaldehyde resin as shell materials.

At present, emulsifiers used for the preparation of PCMs contain anionic surfactants, cationic surfactants, and nonionic surfactants, such as SMA [13, 14], SDBS [15], OP-10 [16], imidazoline [17], and the compound emulsifier [18]. These emulsifiers can reduce Gibbs free energy produced by the mechanical agitation process of emulsion. And the thermal stability and mechanical stability of the emulsion are very important for the core material emulsion in avoiding phase separation phenomenon in the preparation of PCMs $[19,20]$. And emulsifiers have a great influence on the morphology and encapsulation effects of the phase change capsules. Few 
studies about the effects of the different types of emulsifiers on the preparing B-UPCS have been reported. In this study, B-UPCS were synthesized by microencapsulation technology using different types of emulsifiers. In this paper, effects of OP-10 and sodium dodecyl benzene sulfonate (SDBS) in preparation of B-UPCS were discussed in detail. It also offered some useful data for finding a suitable emulsifier or compound emulsifier to get novel PCMs in the future research work.

\section{Experimental}

2.1. Materials. Urea (Yongda Chemical Reagent Co., Ltd., Tianjin, China) and formaldehyde (37\%, Tianli Chemical Reagent Co., Ltd. Tianjin, China) were used to synthesise UF resin. $\beta$-CD (Baishi Chemical Co., Ltd., Tianjin, China) was used as modifier. OP-10 (Guangfu Fine Chemical Research Institute, Tianjin, China) and SDBS (Bodi Chemical Co., Ltd., Tianjin, China) were used as emulsifiers. Resorcin (Kermel Chemical Reagents Co., Ltd., Tianjin, China) and ammonium chloride (Yongda Chemical Reagent Co., Ltd., Tianjin, China) were used as curing agent. Triethanolamine (Hongyan Chemical Reagent Co., Ltd., Tianjin, China) and citric acid (Tianjin Ruijinte Chemical Reagent Co., Ltd., Tianjin, China) were used as $\mathrm{pH}$ regulators. Paraffin (Ruhr Energy Technology Co., Ltd., Hangzhou, China) was used as the core material.

2.2. Preparation of Spherical $\beta$-UPCS. Urea, formaldehyde aqueous solution (37 $\mathrm{wt} \%)$, and distilled water were mixed and the $\mathrm{pH}$ was adjusted to 8-9 with triethanolamine, and then the mixture was stirred at $70^{\circ} \mathrm{C}$ with a stirring rate of $450 \mathrm{rpm}$ for $60 \mathrm{~min}$ to prepare UF precursor solution. $\beta$ $\mathrm{CD}$ was added into the UF precursor solution and mixed in ice bath for $15 \mathrm{~min}$ to obtain the B-CD-UF mixture. The emulsifier, paraffin melt, water, and cyclohexane were dispersed at the high stirring speed for $15 \mathrm{~min}$ to form an oilin-water dispersion. Afterwards, the B-CD-UF mixture, citric acid solution, ammonium chloride, and resorcin were added into the paraffin dispersion with a stirring rate of $500 \mathrm{rpm}$ and kept to $75^{\circ} \mathrm{C}$ for 3 hours. After filtration, washing with distilled water, and drying for $48 \mathrm{~h}$ at room temperature, BUPCS paraffin capsules were obtained.

2.3. Characterization. Fourier transform infrared spectroscopy (FTIR) spectra of B-UPCS were analyzed on a Thermo Fisher Nicolet 6700 FTIR spectrometer (USA) to determine the chemical structure of B-UPCS. The morphology (SEM) of B-UPCS was observed by an FEI QUANTA 200 scanning electronic microscope (USA). Optical micrographs of paraffin emulsions were obtained by a Nikon E200 optical microscope (Japan). Particle sizes of the products were studied by a Brookhaven ZetaPlus zeta potential analyzer (USA). The thermal property measurements were carried out on a TA Q20 differential scanning calorimeter (DSC) (USA) at a heating rate of $5^{\circ} \mathrm{C} / \mathrm{min}$ in the range of 10 to $65^{\circ} \mathrm{C}$ under nitrogen atmosphere.

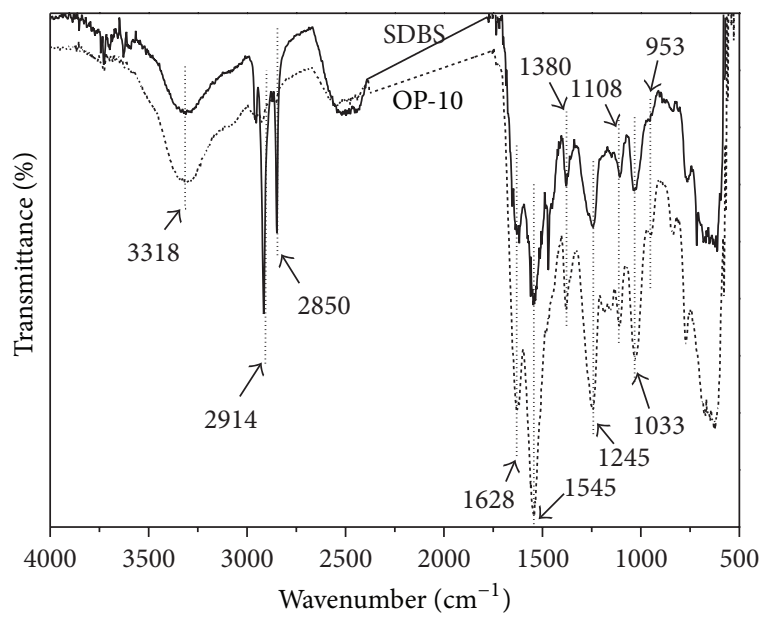

FIGURE 1: FT-IR spectra of B-UPCS with different types of emulsifiers.

2.4. Calculation of Paraffin Content in $\beta$-UPCS. The core material content of B-UPCS directly affects heat storage capacity. The content of the core material can be obtained in different ways. In this study, the paraffin content of $\beta$-UPCS was calculated as follows:

$$
\mathrm{Ca}(\%)=\frac{\Delta H_{\text {microPCM }}}{\Delta H_{\mathrm{PCM}}} \times 100 \%,
$$

where $\Delta H_{\text {microPCM }}$ is enthalpy of $\beta$-UPCS, $\Delta H_{\mathrm{PCM}}$ is enthalpy of paraffin itself $[20,21]$.

\section{Results and Discussion}

3.1. FTIR Spectra of B-UPCS with Different Types of Emulsifiers. FTIR spectra of B-UPCS with different types of emulsifiers are shown in Figure 1. As seen from Figure 1. The peak at $3318 \mathrm{~cm}^{-1}$ corresponded to stretching vibrations of $\mathrm{N}-\mathrm{H}$ and $\mathrm{O}-\mathrm{H}$ [22]. Bands of $2914 \mathrm{~cm}^{-1}$ and $2850 \mathrm{~cm}^{-1}$ can be attributed to $-\mathrm{CH}_{2}$ stretching vibration asymmetric and symmetric adsorption. The peaks around $1628 \mathrm{~cm}^{-1}$, $1545 \mathrm{~cm}^{-1}$ were attributed to stretching vibration of $\mathrm{C}=\mathrm{O}$ and deformation vibration of $\mathrm{CN}, \mathrm{N}-\mathrm{H}[23,24]$. The absorption peak at $1380 \mathrm{~cm}^{-1}$ can be attributed to $\mathrm{C}-\mathrm{H}$ bending vibration and the band at $1245 \mathrm{~cm}^{-1}$ was assigned to $\mathrm{C}-\mathrm{OH}$ inplane stretching vibration. The band $1033 \mathrm{~cm}^{-1}$ corresponded to asymmetric stretching vibration of $\mathrm{C}-\mathrm{O}-\mathrm{C}$ [25]. Thus, in view of the above, it is established that B-UPCS have been synthesized successfully both with OP-10 and SDBS as emulsifier. Comparing the FTIR spectra of B-UPCS with OP10 and SDBS as emulsifier, the emulsifiers had emulsification effects on forming homogeneous paraffin emulsion and there were no significant impacts on the chemical structures of BUPCS.

\subsection{Effects of the Dosage of Emulsifiers on the Average} Diameter of B-UPCS. The relationships among OP-10 dosage, the average particle diameter of B-UPCS, and the particle size of paraffin emulsion are shown in Figure 2. As seen 


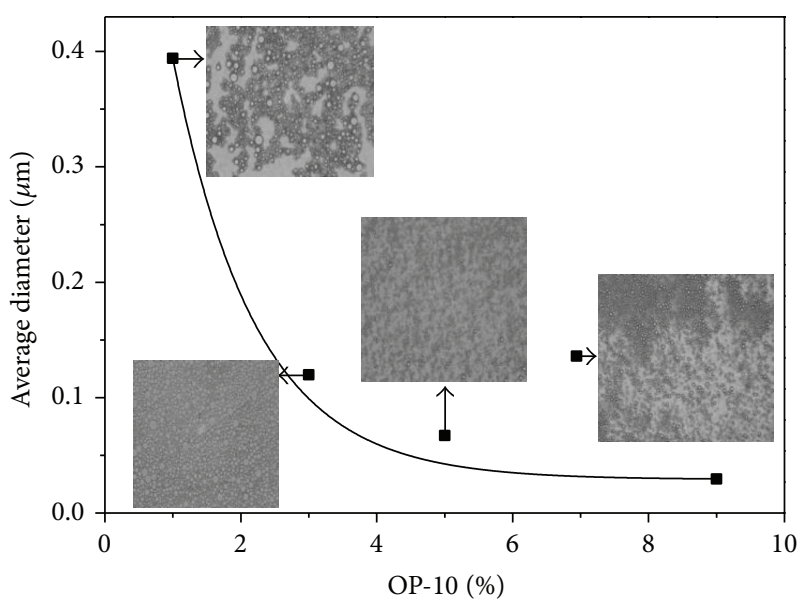

FIGURE 2: Effect of the dosage of OP-10 on average particle diameter of B-UPCS and the optical micrographs of paraffin emulsion.

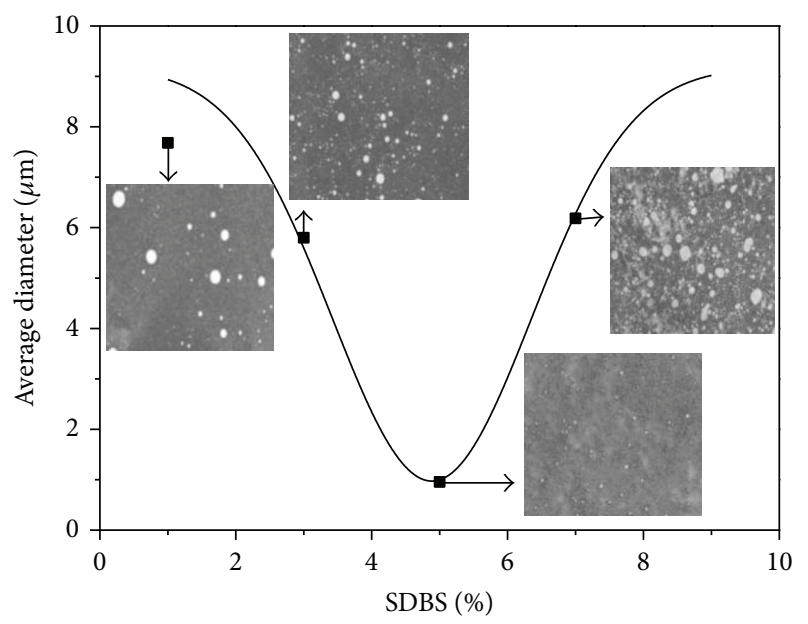

FIGURE 3: Effects of the dosage of SDBS on average particle diameter of B-UPCS and the optical micrographs of paraffin emulsion.

from Figure 2, the particle sizes of B-UPCS and paraffin emulsion were decreased rapidly with the increase of the OP10 dosage. When the dosage of OP- 10 reached $3 \%$ to $7 \%$, the particle sizes of B-UPCS were approximately $0.1 \mu \mathrm{m}$. The result indicates that OP-10 adhered to the droplets surface and brought a balance state between the prepolymers and the curing resin. When the dosage of OP-10 reached 7\% to $9 \%$, the average diameter of B-UPCS remained unchanged with the increase of OP-10. At this condition, the effects of OP-10 on the average particle size of B-UPCS and paraffin emulsion were not remarkable. The drastic self-polymerization reaction between methylolureas and $\beta$-CD did not occur in the end due to the fact that the main composition of OP10 is polyoxyethylene alkylphenol with a high Hydrophilelipophile balance number (HLB) value of 14.5. OP-10 had a strong emulsifying ability for paraffin to form a uniform, stable, and well-dispersed emulsion solution because of the nonpolar groups in OP-10 [26]. Thus, the dosage of emulsifier is a key factor for the droplet size of paraffin-water emulsion

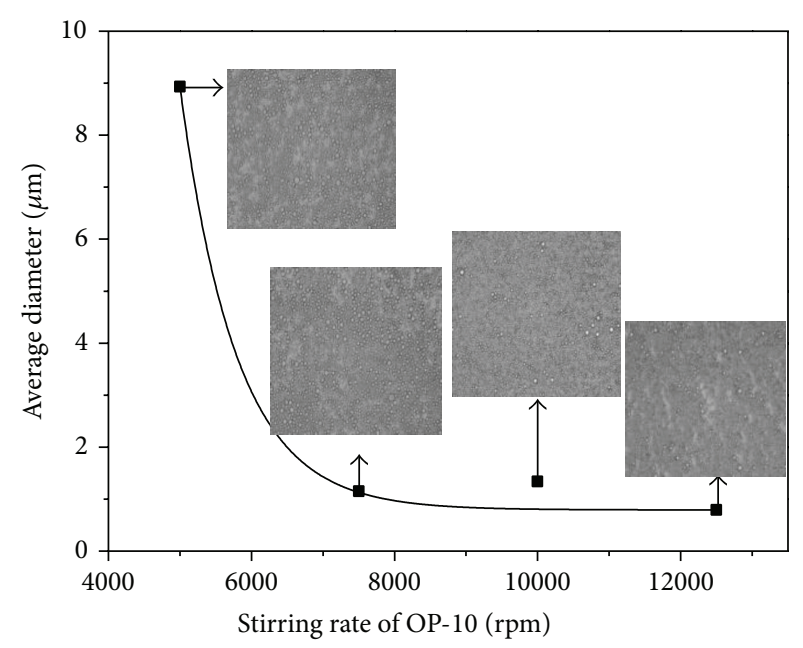

FIGURE 4: Effects of stirring rate of OP-10 on average particle diameter of B-UPCS and the optical micrographs of paraffin emulsion.

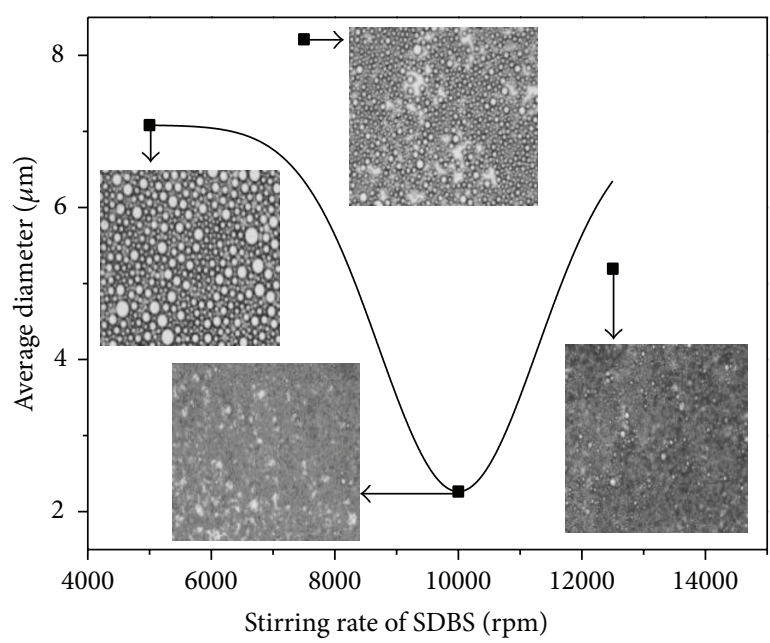

FIGURE 5: Effects of stirring rate of SDBS on average particle diameter of B-UPCS and the optical micrographs of paraffin emulsion.

and the uniformity of the core material. Based on the above results, the optimum dosage of OP-10 was $3 \%$ to $7 \%$.

The relationships among SDBS dosage, the average particle diameter of B-UPCS, and the particle size of paraffin emulsion are shown in Figure 3. With the increase of SDBS dosage, the average particle diameter of B-UPCS presented sudden decrease firstly and later increase trend. When the dosage of SDBS was $1 \%$ to $5 \%$, the average particle diameter of B-UPCS decreased rapidly because the surface charges of SDBS reduced oil-water interfacial tension and Gibbs free energy of paraffin emulsion solution. With the increase of the dosage of SDBS, a homogeneous paraffin emulsion solution was gradually formed with a good resistance to temperature and the mechanical agitation. When the amount of SDBS reached more than $7 \%$, the particle diameter of B-UPCS increased rapidly, and the probable reason for this phenomenon was the violent curing reaction or the sensitivity of SDBS for temperature or mechanical agitation 


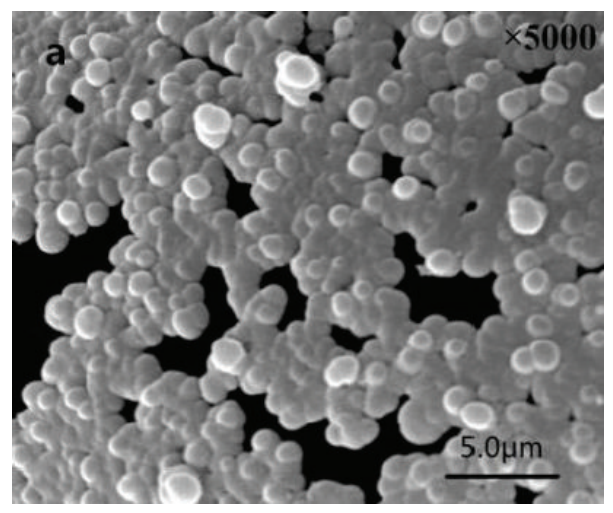

(a)

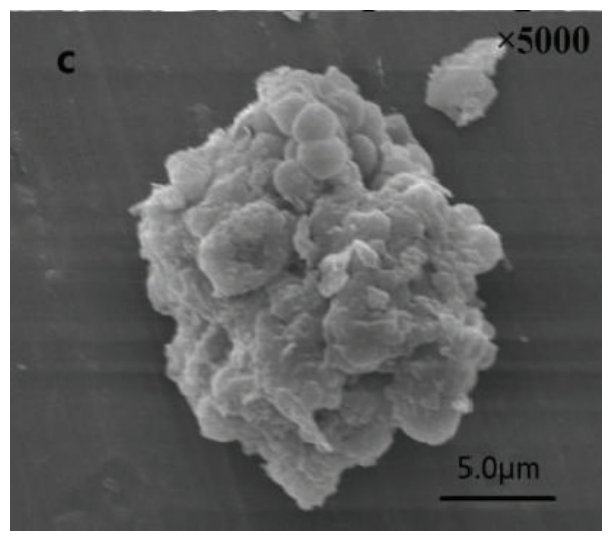

(c)

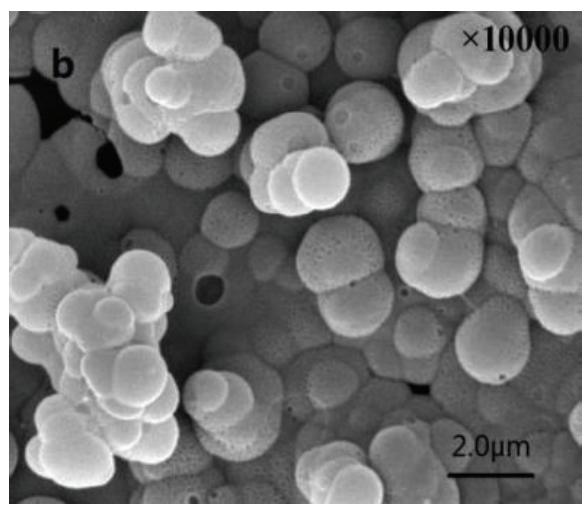

(b)

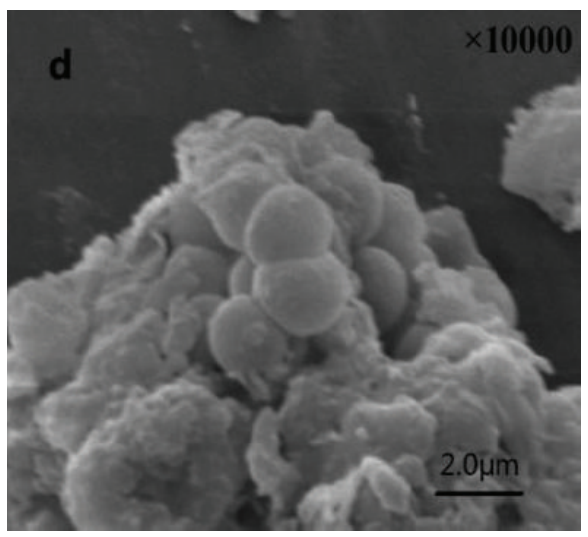

(d)

FIGURE 6: SEM images of B-UPCS prepared with different emulsifiers. The dosage of emulsifiers was $0.3 \mathrm{~g}$, and the stirring rate of paraffin was $10000 \mathrm{rpm}$.

to result in phase separation phenomenon, thereby generating a large block of B-UPCS [27]. Thus, the optimum dosage of SDBS was $4 \%$ to $6 \%$.

\subsection{Effects of the Stirring Rate of Emulsifiers on Average} Diameter of B-UPCS. The dosage of emulsifier is a key factor on dispersing the core material of B-UPCS. It had great influences on the preparing PCMs with uniform particle size. Effects of stirring rate of OP-10 on average particle diameter of B-UPCS are shown in Figure 4. With the stirring rate of OP-10 increasing, the average particle diameter of B-UPCS and the size of paraffin droplets decreased rapidly (Figure 4). When the stirring rate reached $10000 \mathrm{rpm}$, the core material began to form uniform and stable emulsion solution, and the average particle diameter of B-UPCS remained unchanged. And this trend is in accordance with the literature [28]. Thus, OP10 had a strong stability of temperature and the mechanical agitation and the phase separation phenomenon was difficult to occur. As a result, OP-10 can be used as an emulsifier with a broader range of the stirring rate. In this study, the optimum stirring rate of OP-10 was $7500 \sim 12500 \mathrm{rpm}$ with desired emulsion effects.

Effects of stirring rate of SDBS on average particle diameter of B-UPCS are shown in Figure 5. As seen from Figure 5, the optimum stirring rate of SDBS as emulsifier was
$10000 \mathrm{rpm}$. Phase separation phenomenon occurred when the stirring speed increased to $12500 \mathrm{rpm}$, resulting in a sharp increase of the particle diameter. The results were consistent with the ones of the optical microscope. Therefore, the stirring rate of SDBS was $10000 \mathrm{rpm}$.

\subsection{Effects of Different Types of Emulsifiers on Microstructure} of B-UPCS. SEM images of B-UPCS with OP-10 (Figures 6(a) and 6(b)) and SDBS (Figures 6(c) and 6(d)) as emulsifier are shown in Figure 6. It can be seen from Figures 6(a) and 6(b) that B-UPCS prepared by in situ polymerization were spherical. The capsule particles were composed of many small particles depositing on the surface of B-UPCS, only a few collapse and breakage phenomenon in B-UPCS. B-UPCS had uniform particle. This result indicated that OP-10 had good emulsion effects on the dispersion of the core material, and the result was consistent with the results above.

As seen from Figures 6(c) and 6(d), a large area of adhesion and accumulation existed on the surfaces of B-UPCS. The probable reason is that the prepolymer attached to the surface of the paraffin droplets and cured violently. Besides, it is obvious that B-UPCS consist of many small spherical capsules with tightness surface, which is more suitable for providing good encapsulation effects and almost no leakages for the core material. 


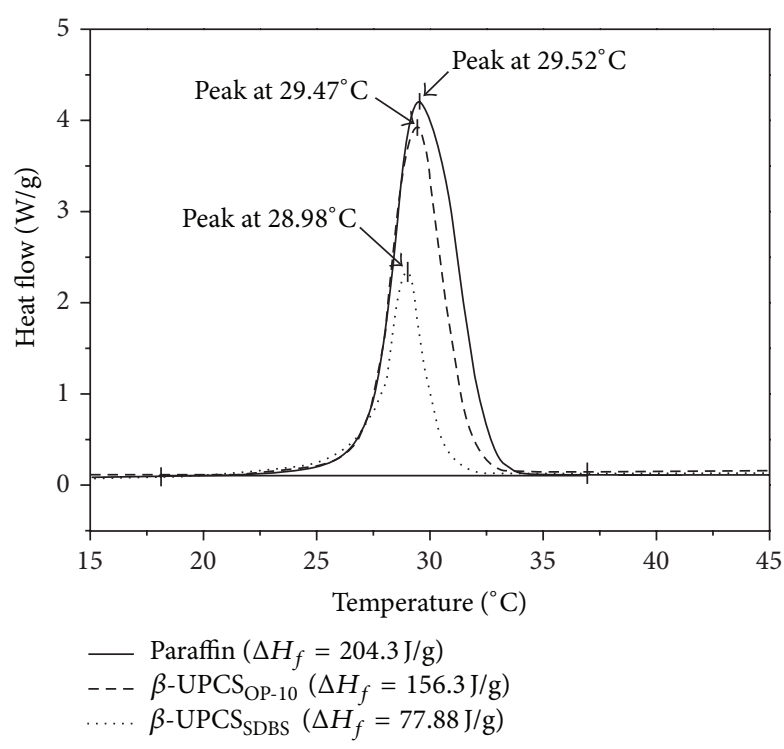

FIGURE 7: DSC curves of B-UPCS and paraffin.

3.5. DSC Curves of B-UPCS. The DSC curves of B-UPCS with $\mathrm{OP}-10$ and SDBS as emulsifier are shown in Figure 7. The phase change enthalpy of B-UPCS with OP-10 as emulsifier was higher than the one with SDBD as emulsifier. Most of BUPCS prepared with OP-10 as emulsifier were spherical with good barrier properties and most paraffin was wrapped into B-UPCS. For SDBS as emulsifier, too violent curing reaction resulted in only part of paraffin wrapped in B-UPCS. The main reason is that the poor dispersion capacity of SDBS led to the self-polymerization reaction of monomer. As a result, the enthalpy of B-UPCS with SDBS as emulsifier was low. This result was consisted with SEM. As seen from Figure 7, the phase transition temperatures of B-UPCS were different form pure paraffin, probably due to the interactions between the core and shell material in B-UPCS [21]. The results were the same as our previous literatures [29-31].

3.6. Effects of Different Types of Emulsifiers on the Paraffin Content. Effects of the dosage of OP-10 and SDBS as emulsifiers on the content of paraffin in B-UPCSare shown in Figure 8. And in this paper, the paraffin content of $\beta$-UPCS was calculated according to (1). With the dosage of OP-10 increasing, the content of paraffin in B-UPCS increased at first and then decreased slightly. When the dosage of OP10 reached $3 \%$ to $7 \%$, the content of paraffin was about $78 \%$. When the dosage of OP-10 reaches $9 \%$, the content of paraffin in B-UPCS tended to decrease. When the dosage of OP-10 was low, it helped to reduce Gibbs free energy, caused by temperature and mechanical stirring, and promoted to help the monomer adhere to the droplets surface of paraffin emulsion and formed the core-shell structure capsules [31]. However, when the dosage of OP-10 continued to increase, the paraffinwater phase was separated and the polycondensation reaction occurred among the monomers of the shell material. As a result, the paraffin in B-UPCS declined. And, with SDBS as emulsifier, the content of paraffin in $\beta$-UPCS was increased

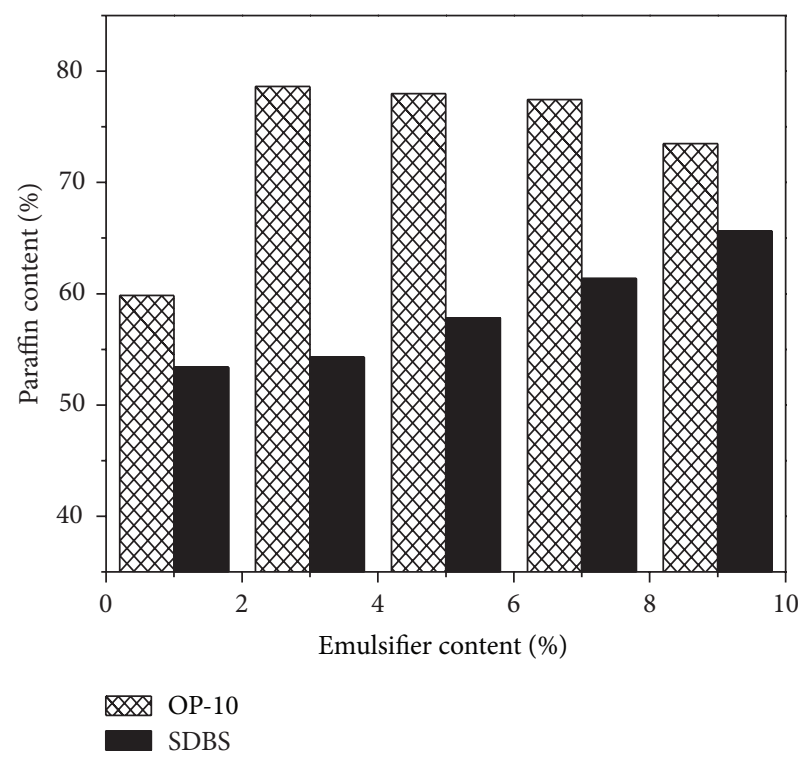

FIGURE 8: Effects of the emulsifier dosage on the content of paraffin in B-UPCS.

slowly with the increase of SDBS dosage. This was perhaps because SDBS was ionic surfactant and it has little influences on wrapping paraffin in water-oil phase. However, at the same dosage of OP-10 as emulsifier, the paraffin content in B-UPCS was significantly higher because OP-10 has better emulsion effect than SDBS on dispersing the core material. Hence, it could continuously promote prepolymers to curing on the droplet surface of paraffin emulsion by crosslinked reaction and formed well-encapsulated B-UPCS.

\section{Conclusion}

In this study, the effects of two types of emulsifiers (OP10 , SDBS) on paraffin content average particle size and microstructure of B-UPCS were discussed by changing the stirring rate and the dosage of emulsifiers. The experimental results showed that when the dosage of OP-10 reached $4 \%$ to $7 \%$ and the stirring rate was $7500 \sim 12500 \mathrm{rpm}$, the spherical particle diameter of B-UPCS was uniform and the paraffin content was $78 \%$, and the enthalpy of B-UPCS can reach $161.0 \mathrm{~J} / \mathrm{g}$. When the dosage of SDBS reached more than $7 \%$ or the stirring rate increased to $12500 \mathrm{rpm}$, demulsification phenomenon of paraffin emulsion occurred, led to a rapid curing reaction. Comparing with SDBS as emulsifier, OP-10 had good emulsion results with a high content of paraffin, uniform particle size, and good dispersion effects. However, there were some individual damaged capsules with OP-10 as emulsifier. In the following study, we will explore different types of compound surfactants for the preparation of the core-shell energy storage materials.

\section{Conflict of Interests}

The authors declare that there is no conflict of interests regarding the publication of this paper. 


\section{Acknowledgments}

This work was supported financially by the National Natural Science Foundation of China (no. 31000277) and the Central University Basic Scientific Research Project of China (no. 2572014DB01).

\section{References}

[1] V. A. A. Raj and R. Velraj, "Review on free cooling of buildings using phase change materials," Renewable and Sustainable Energy Reviews, vol. 14, no. 9, pp. 2819-2829, 2010.

[2] X. Hu, Z. Huang, and Y. Zhang, "Preparation of CMC-modified melamine resin spherical nano-phase change energy storage materials," Carbohydrate Polymers, vol. 101, pp. 83-88, 2014.

[3] A. Malfliet, G. Deferme, L. Stappers, and J. Fransaer, "Synthesis and characterization of composite coatings for thermal actuation," Journal of the Electrochemical Society, vol. 154, no. 1, pp. D50-D56, 2007.

[4] H. Zhang, X. Wang, and D. Wu, "Silica encapsulation of n-octadecane via sol-gel process: a novel microencapsulated phase-change material with enhanced thermal conductivity and performance," Journal of Colloid and Interface Science, vol. 343, no. 1, pp. 246-255, 2010.

[5] M. N. A. Hawlader, M. S. Uddin, and M. M. Khin, "Microencapsulated PCM thermal-energy storage system," Applied Energy, vol. 74, no. 1-2, pp. 195-202, 2003.

[6] J.-F. Su, X.-Y. Wang, and H. Dong, "Influence of temperature on the deformation behaviors of melamine-formaldehyde microcapsules containing phase change material," Materials Letters, vol. 84, pp. 158-161, 2012.

[7] H. Zhang and X. Wang, "Fabrication and performances of microencapsulated phase change materials based on n-octadecane core and resorcinol-modified melamine-formal-dehyde shell," Colloids and Surfaces A, vol. 332, no. 2-3, pp. 129-138, 2009.

[8] Z. Xue and J. Li, "Preparation and characterization of microencapsulated phase change liquid paraffin," The Chinese Journal of Process Engineering, vol. 12, no. 2, pp. 293-301, 2012.

[9] K. Sun, B. Zhang, F. Yan, G. Yin, and Y. Cui, "Preparation and characterization of microcapsule with paraffin phase change materials for energy storage," Chemical Industry and Engineering Progress, vol. 30, no. 12, pp. 2676-2678, 2011.

[10] Z. Jin, Y. Wang, J. Liu, and Z. Yang, "Synthesis and properties of paraffin capsules as phase change materials," Polymer, vol. 49, no. 12, pp. 2903-2910, 2008.

[11] M. Delgado, A. Lázaro, J. Mazo, and B. Zalba, "Review on phase change material emulsions and microencapsulated phase change material slurries: materials, heat transfer studies and applications," Renewable and Sustainable Energy Reviews, vol. 16, no. 1, pp. 253-273, 2012.

[12] C. Fan and X. Zhou, "Influence of operating conditions on the surface morphology of microcapsules prepared by in situ polymerization," Colloids and Surfaces A, vol. 363, no. 1-3, pp. 4955, 2010.

[13] X.-L. Ren, L.-X. Wang, L. Ren, and J.-F. Su, "Preparation of phase change materials microcapsules by in-situ polymerization," Journal of Functional Materials, vol. 36, no. 11, pp. 17221727, 2005.

[14] W. Li, X.-X. Zhang, X.-C. Wang, and J.-J. Niu, "Preparation and characterization of microencapsulated phase change material with low remnant formaldehyde content," Materials Chemistry and Physics, vol. 106, no. 2-3, pp. 437-442, 2007.

[15] L. Yuan, G.-Z. Liang, J.-Q. Xie, J. Guo, and L. Li, “Thermal stability of microencapsulated epoxy resins with poly(urea-formaldehyde)," Polymer Degradation and Stability, vol. 91, no. 10, pp. 2300-2306, 2006.

[16] Q. Lin, D. zhu, J. Li, F. Luo, and W. Zhou, "Preparation and phase change properties of paraffin microcapsules," Fine Chemicals, vol. 28, no. 1, pp. 10-13, 2011.

[17] X. Liu, S. Wang, and H. Liu, "Preparation and characteristics of paraffin microencapsulated in melamine-urea-formaldehyde by in-situ polymerization," Journal of Chemical Industry and Engineering, vol. 57, no. 12, pp. 2991-2996, 2006.

[18] Y. Yan, H. Zhang, and J. Liu, "Preparation and characteristics of microencapsulated phase change materials," Materials Review, vol. 23, no. 2, pp. 49-51, 2009.

[19] F. Tiarks, K. Landfester, and M. Antonietti, "Preparation of polymeric nanocapsules by miniemulsion polymerization," Langmuir, vol. 17, no. 3, pp. 908-918, 2001.

[20] A. Sari, C. Alkan, and A. Karaipekli, "Preparation, characterization and thermal properties of PMMA/n-heptadecane microcapsules as novel solid-liquid microPCM for thermal energy storage," Applied Energy, vol. 87, no. 5, pp. 1529-1534, 2010.

[21] L. Pan, Q. Tao, S. Zhang et al., "Preparation, characterization and thermal properties of micro-encapsulated phase change materials," Solar Energy Materials and Solar Cells, vol. 98, pp. 66-70, 2012.

[22] G. Fang, H. Li, F. Yang, X. Liu, and S. Wu, "Preparation and characterization of nano-encapsulated n-tetradecane as phase change material for thermal energy storage," Chemical Engineering Journal, vol. 153, no. 1-3, pp. 217-221, 2009.

[23] H. Li, Research on surface modification of PUF microcapsules and self-healing performance for epoxy [Ph.D. thesis], Harbin Institute of Technology, Harbin, China, 2010.

[24] J.-Q. Xie, F.-M. Feng, W. Hu, H.-J. Mao, H. Yan, and C. Li, "Preparation of a microcapsule containing the paraffin as the core material by in-situ polymerization method without prepolymerization and capability of the microcapsule," Journal of Functional Materials, vol. 39, no. 2, pp. 293-296, 2008.

[25] L. Yuan, G. Liang, J. Xie, L. Li, and J. Guo, "Preparation and characterization of poly(urea-formaldehyde) microcapsules filled with epoxy resins," Polymer, vol. 47, no. 15, pp. 5338-5349, 2006.

[26] X. Hu, Z. Huang, X. Yu, and B. Li, "Preparation and thermal energy storage of carboxymethyl cellulose-modified nanocapsules," BioEnergy Research, vol. 6, no. 4, pp. 1135-1141, 2013.

[27] M. Alcoutlabi and G. B. McKenna, "Effects of confinement on material behaviour at the nanometre size scale," Journal of Physics Condensed Matter, vol. 17, no. 15, pp. R461-R524, 2005.

[28] Z.-H. Chen, F. Yu, X.-R. Zeng, and Z.-G. Zhang, "Preparation, characterization and thermal properties of nanocapsules containing phase change material $\mathrm{n}$-dodecanol by miniemulsion polymerization with polymerizable emulsifier," Applied Energy, vol. 91, no. 1, pp. 7-12, 2012.

[29] C. Alkan, A. Sari, and A. Karaipekli, "Preparation, thermal properties and thermal reliability of microencapsulated n-eicosane as novel phase change material for thermal energy storage," Energy Conversion and Management, vol. 52, no. 1, pp. 687692, 2011.

[30] G. Fang, Z. Chen, and H. Li, "Synthesis and properties of microencapsulated paraffin composites with $\mathrm{SiO}_{2}$ shell as thermal energy storage materials," Chemical Engineering Journal, vol. 163, no. 1-2, pp. 154-159, 2010. 
[31] X. L. Shan, J. P. Wang, X. X. Zhang, and X. C. Wang, "Formaldexhyde-free and thermal resistant microcapsules containing noctadecane," Thermochimica Acta, vol. 494, no. 1-2, pp. 104-109, 2009. 

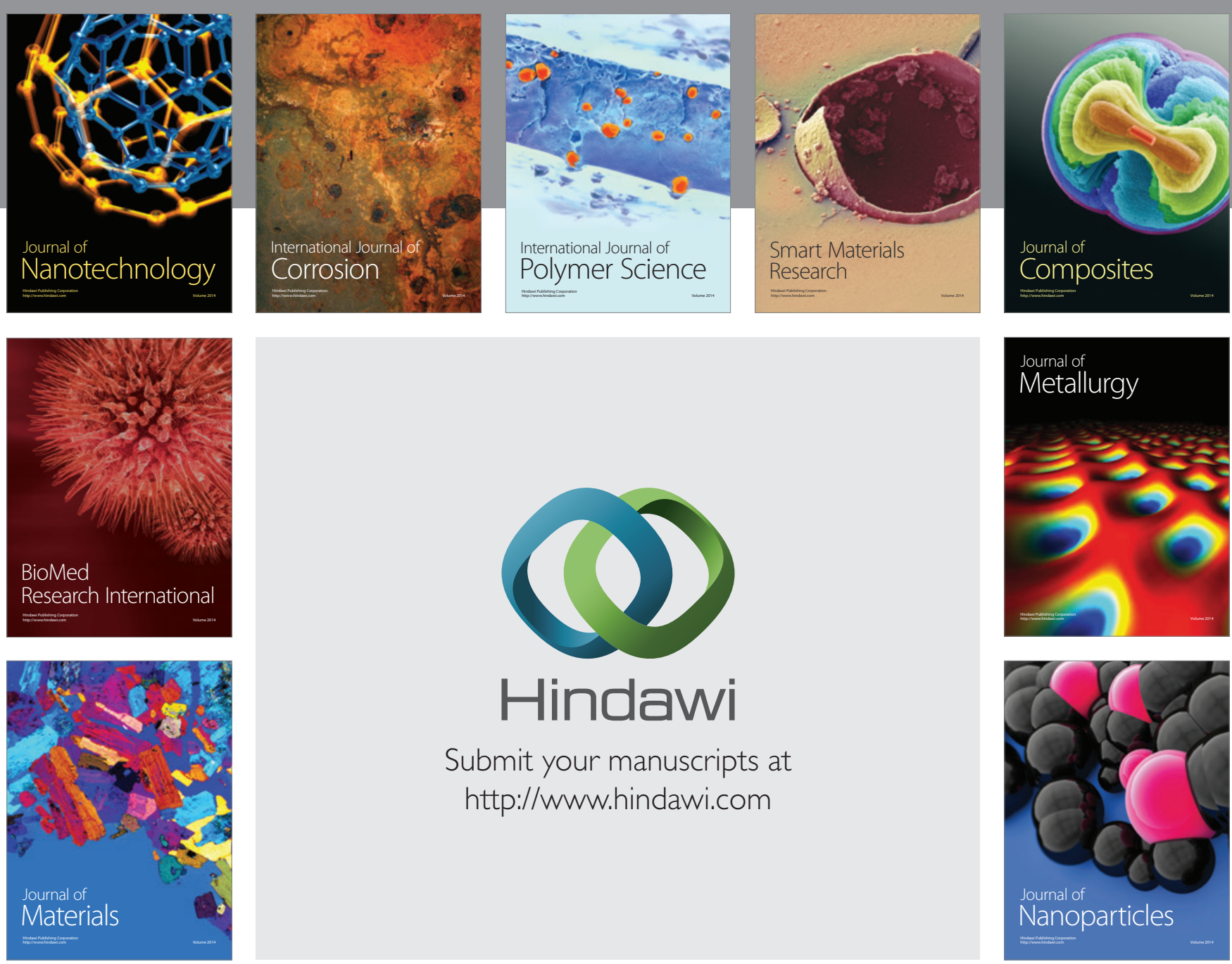

Submit your manuscripts at http://www.hindawi.com
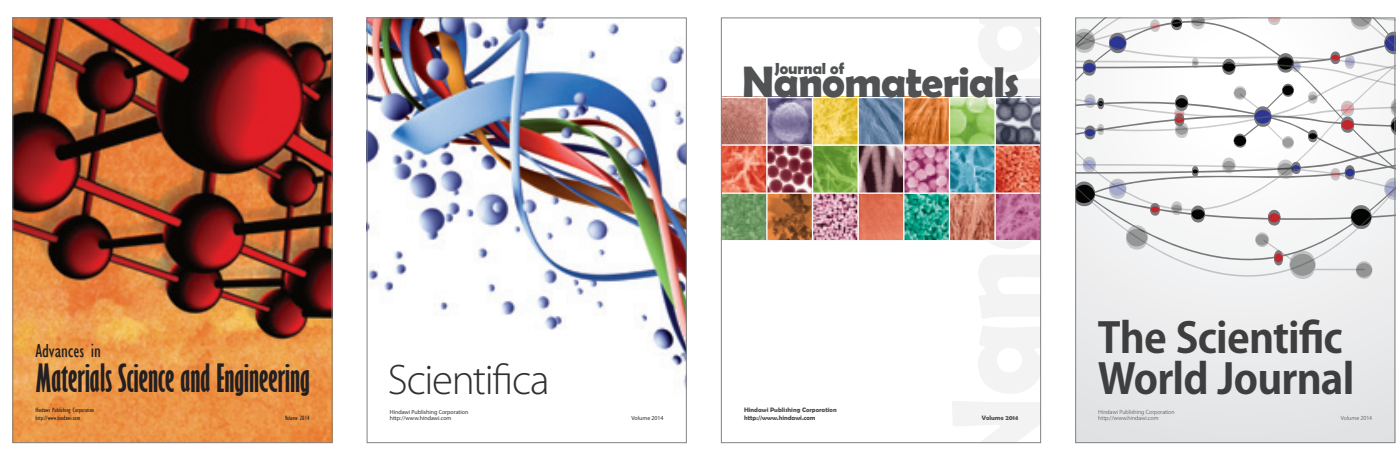

\section{The Scientific World Journal}
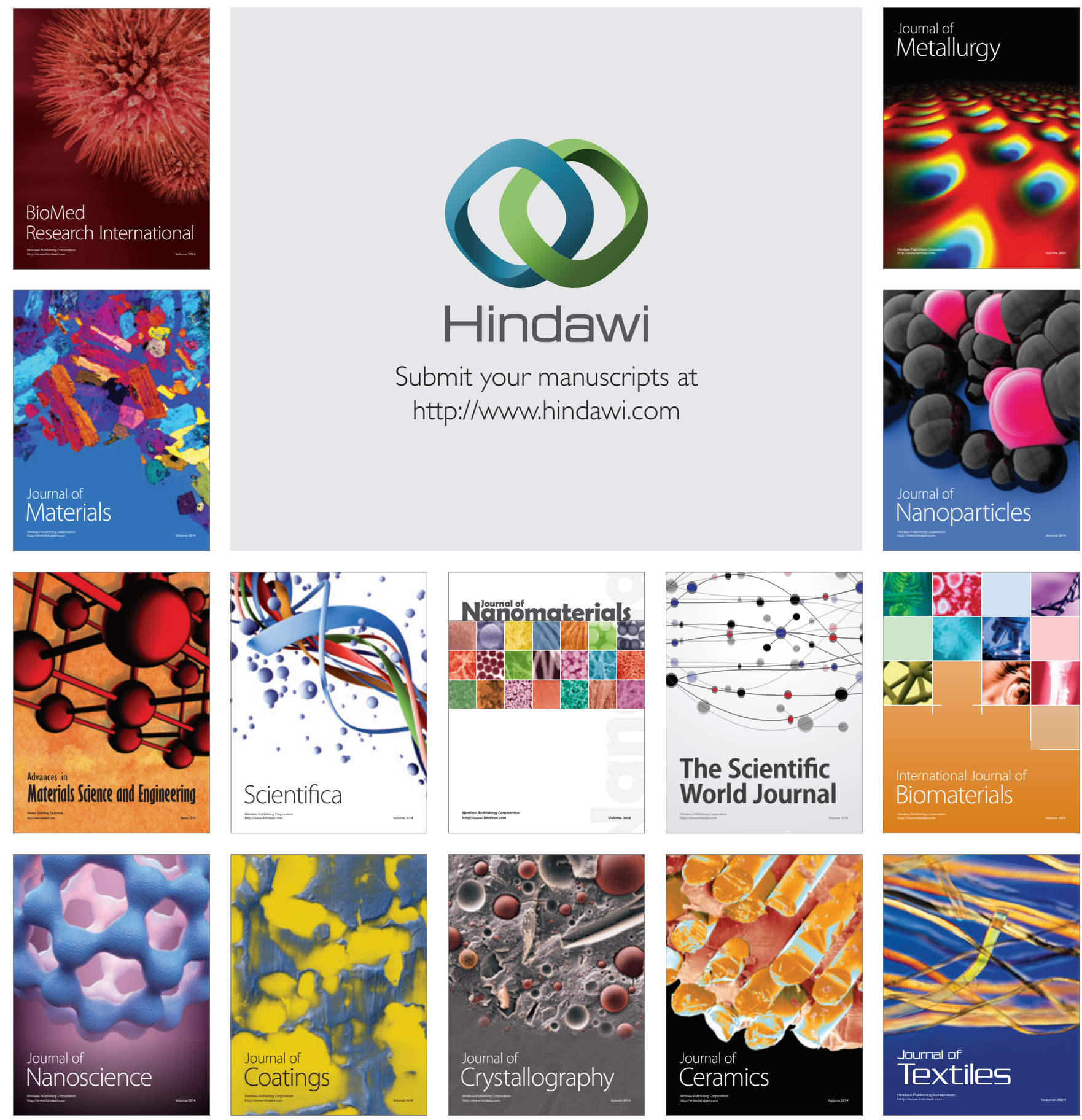